\title{
Unification of Design Decisions for Elements of Products Described by Structured Multiple Modules
}

\author{
Boris Bazrov ${ }^{1}$, Mikhail Kheifetz ${ }^{2}$, Nikolay Popok ${ }^{3}$ \\ ${ }^{1}$ Mechanical Engineering Research Institute of the Russian Academy of Sciences, Russian Federation \\ ${ }^{2}$ Open Joint Stock Company "NPO Center" of The National Academy of Sciences of Belarus, \\ Republic of Belarus \\ ${ }^{3}$ Polotsk State University, Republic of Belarus
}

\begin{abstract}
The shortcomings of the traditional description of the engineering product are shown. It is proposed to represent the design of the product and its details with a structured set of corresponding modules, in the form of a graph of a hierarchical structure. The characteristics of the structure of the product graph are considered: the number of levels, nodes, branches. The description of the structures of products by a hierarchical graph at the first level makes it possible to identify functional technological modules of the products and, on their basis, to construct a unified classification of products as objects of exploitation. Representation of parts by a set of modules allows you to identify modules based, working and connecting surfaces and on their basis to build a single classification of parts, focused on different stages of the product life cycle. The presence of a unified methodological base allows you to manage the development of product designs, minimize duplication in the creation of new designs and effectively develop technologies for their manufacture.
\end{abstract}

\section{Introduction}

The continuity of design-technological and organizational-economic decisions in the preparation of production, exploitation and maintenance, control and management are based on a common information space of a virtual enterprise that integrates distributed databases [1-3]. Therefore, the development of a rational information model is the foundation of the complex computerization of design, technological and metrological preparation of production [4 - 6]. Design and manufacture, exploitation and maintenance of products require, above all, an assessment of the reliability, adaptability and efficiency of pre-production [7-9]. Methods of automating pre-production, widely used in practice, are divided into three large groups: typical parts and technological processes, applications of component parts and structural parametrization [10 - 13].

The lack of flexibility in the method of typical parts and technical processes, the high complexity of the method of applications is due to the one-level organization of the description of structures, objects and automation processes. The reliability of the structuralparametric method is ensured by the construction of structures with a small number of connections, and the universality is ensured by the presence of adaptive structuraltechnological components. The complexity of the method is due to the classification and unification of structural components and technological functions relative to the selected range of parts. Classification and subsequent unification according to constructive- 
technological and operational-functional features allow reducing the number of parameters, simplifying the structuring of the objects and processes under consideration [1-3].

\section{Description of the product design with a variety of modules}

Engineering products are distinguished by a huge, continuously growing variety. Therefore, in these conditions, it is important to build a single database of product designs and their parts. The presence of a single base allows you to manage the development of product designs, minimize duplication in the creation of new designs and effectively develop technologies for their manufacture $[14,15]$. To solve these problems, it is necessary, first, to obtain information on the characteristics of product designs (PD). The traditional description of the PD includes an assembly drawing, detail drawings, an explanatory note and does not meet modern requirements. In the drawings, significant parts of the numerous characteristics are presented in an implicit, weakly formalized form. Therefore, in the drawing of the part does not indicate the number of surfaces, their functional purpose and the connection between them. To eliminate the noted deficiencies, a description of the PD is proposed using a set of modules $[1,16]$.

As PD modules are take the module of a group of parts (MP) of a product and the module of a group of surfaces of a part - the module of surfaces (MS). The unification of parts in MP and surfaces of a detail in MS is determined by the stage of the product's life cycle, since at different stages MP and MS perform different roles and are represented by a different composition, respectively, of parts and surfaces.

Thus, the product can be represented by a set of MP, and if all the details to replace the sets of MS, then PD can be represented by a set of MS. The modular structure of PD can be described by a graph of a hierarchical structure, the top of which is the basic detail. For example, in a metal cutting machine, the base part is a bed.

Graph PD is constructed as follows. First, the basic part of the product is taken as the top of the graph, then the elements of the PD (MP or parts) installed on the base part are determined, then the elements installed on the elements of the previous level, etc. are determined. to the last item.

Now, to describe the PD, we use the characteristics of the graph - numbers: elements, levels, nodes, nodes at each level, and branches. The edges of the graph indicate the coordinating dimensions connecting the sets of parts bases, which are the main auxiliary bases. The main bearing surfaces are the surfaces by which the part is installed into the product, and the auxiliary based surfaces are the surfaces on which other parts are mounted on the part. The graph node carries information about the characteristics of the element PD. For example, if a node reflects a detail, then its mass, overall dimensions, material, etc. are indicated. It is advisable to reflect the characteristics of the nodes in tabular form.

Depending on the level of detail of the description of the PD, graphs of three levels are built: MP and parts not included in their composition (first level), details (second level), MS (third level).

On the first level graph, the MP and the details that are not included in them are the nodes of the graph. At the graph of the second level, as the nodes act as details. At the graph of the third level as the nodes are the MS. The second level graph is constructed by replacing each MP in the first level column with their detail graphs. The third level graph is constructed by replacing each part in the second level column with their MS graphs.

Graphs in the foreseeable concentrated form provide information about the structure of the PD: the composition of the elements, its structure, dimensional, accuracy, strength, hardness and other relationships between the elements of $\mathrm{PD}$, affecting the formation of output indicators of PD. 
Consider the description of PD as an object of operation. The product is designed to perform the operation, therefore, all PD modules carry the corresponding functional load.

Similarly, the surface of the part is intended to perform, respectively, service functions. These modules are denoted MPF and MSF.

According to the functional feature, MPF and MSF should be divided into functional technological modules (MFT, MST) and service functional modules (MFS, MSS): MFTthe part of the PD with which the PD performs its official function; MFS - part of the PD that ensures that the MFT fulfills its purpose; MST - a combination of surfaces of a part, with the help of which the part performs the corresponding service function; MSS- a combination of surfaces with the help of which MST fulfills its purpose.

Combining parts in MPF and combining the surfaces of the part in the MSF on a functional basis ensures the unambiguity of their definitions. For example, in the case of a lathe a spindle assembly with a chuck (MFT1) for setting the workpiece and a tool holder (MFT2) for installing the tool act as an MFT.

To ensure the law of relative motion of the MFT1 and MFT2, the MFSs are the gearbox (MFS1), the gearbox (MFS2), the engine (MFS3).

In the case of an MTF vehicle, a body for placing a load (MFT1) and a chassis (MFT2) to ensure the movement of the vehicle are used, and as an MFS are an engine (MFS1), a transmission (MFS2) and other devices that ensure that the MFT performs its functions.

As for the part, it is generally intended for the product to perform the workflow and to install other parts on it. Therefore, the base modules and work surface modules act as MST for the part. For example, in a gear wheel, a set of side surfaces of the teeth for transmitting torque and a second MST - a set of bases: an end, a hole, and a side surface of the key groove for mounting a gear in the product act as one MST. To fulfill its official purpose, the listed MST are combined into a part with the help of connecting surfaces united in MSS.

\section{Classification of functional technology modules}

For the presentation of the details of the MSF set, the following classification is proposed. By service, all MSF are divided into three classes: basing (MSB), working (MSW), connecting (MSC). Such a division of the MSF gives them an unambiguous definition and is the main difference and advantage of this classification.

The next step in the development of the classification of the MSF is the division of each class into subclasses, groups, subgroups. Therefore, further each MSF class should be subdivided according to constructive and geometrical features.

In Fig.1. a classification of the types of MSF is given, from which it follows that it contains 26 types of MSF, of which 14 are MSBs and six types of MSW and MSC each.

Their examples are shown in Fig.2.

Fig. 3, a shows the drawing of the part in a modular design, where all its surfaces are combined into groups and corresponding modules.

Count MSF details are constructed as follows (Fig. 3, b).

For the top of the graph we take the MSB, which acts as a set of main bases for the part. At the second level are located the MSF, the design base of which is the first level MSF. At the next level, there are MSFs, the design base of which is the MPF of the previous level, and this continues to the last MSF details. 


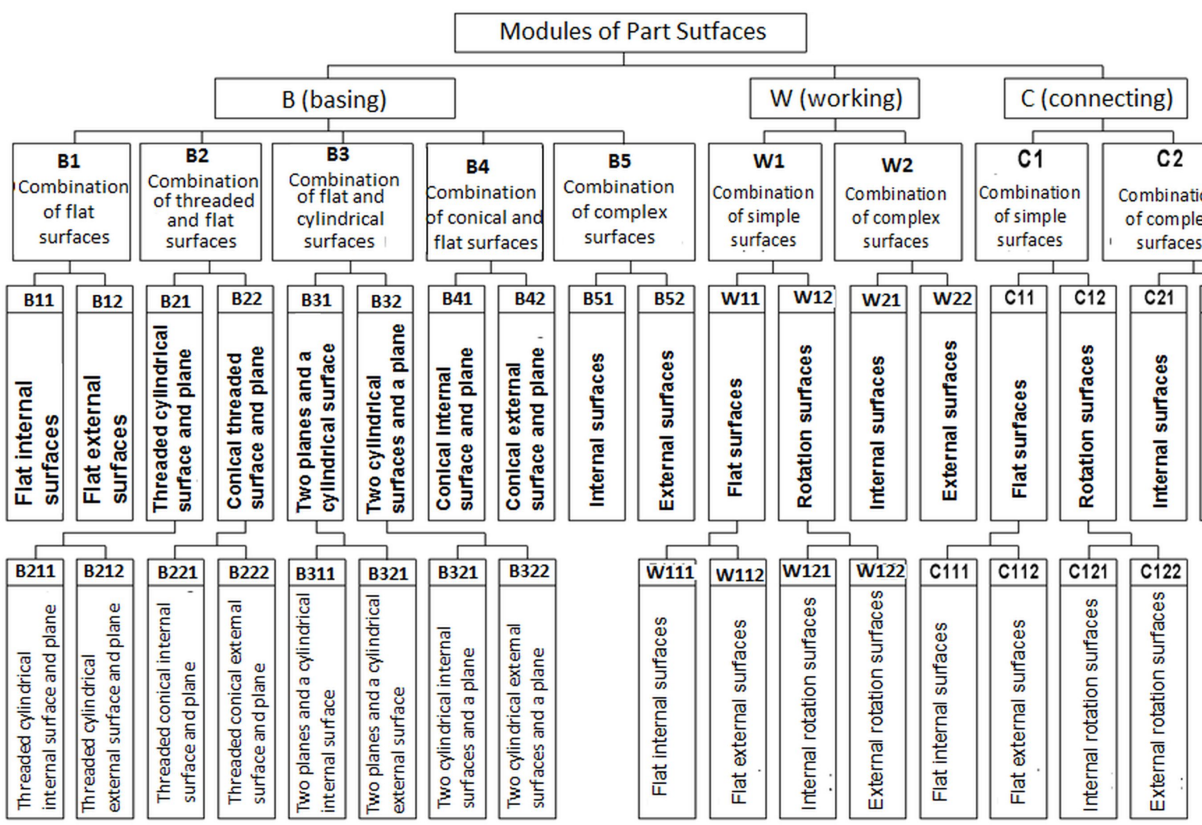

Fig. 1 Classification of MSP

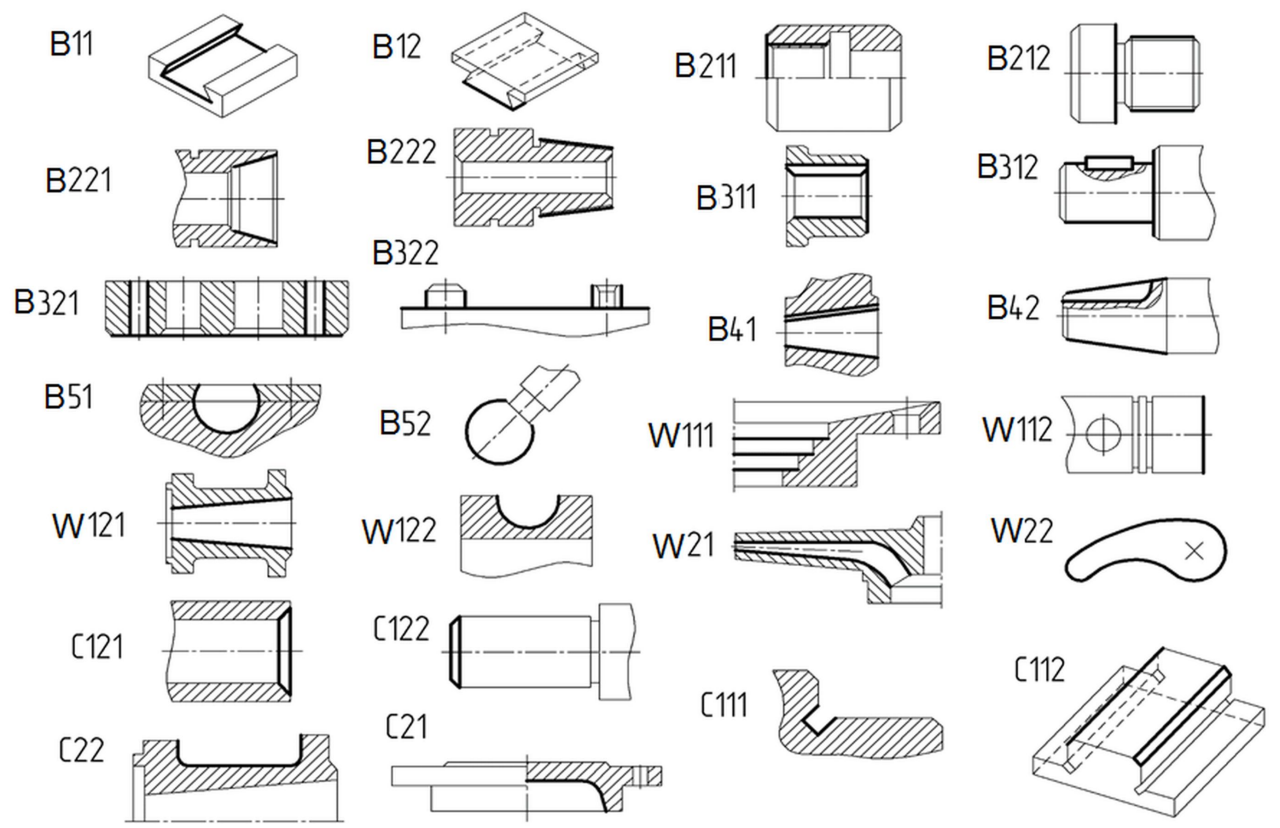

Fig. 2 Examples of constructive design types of MSF 


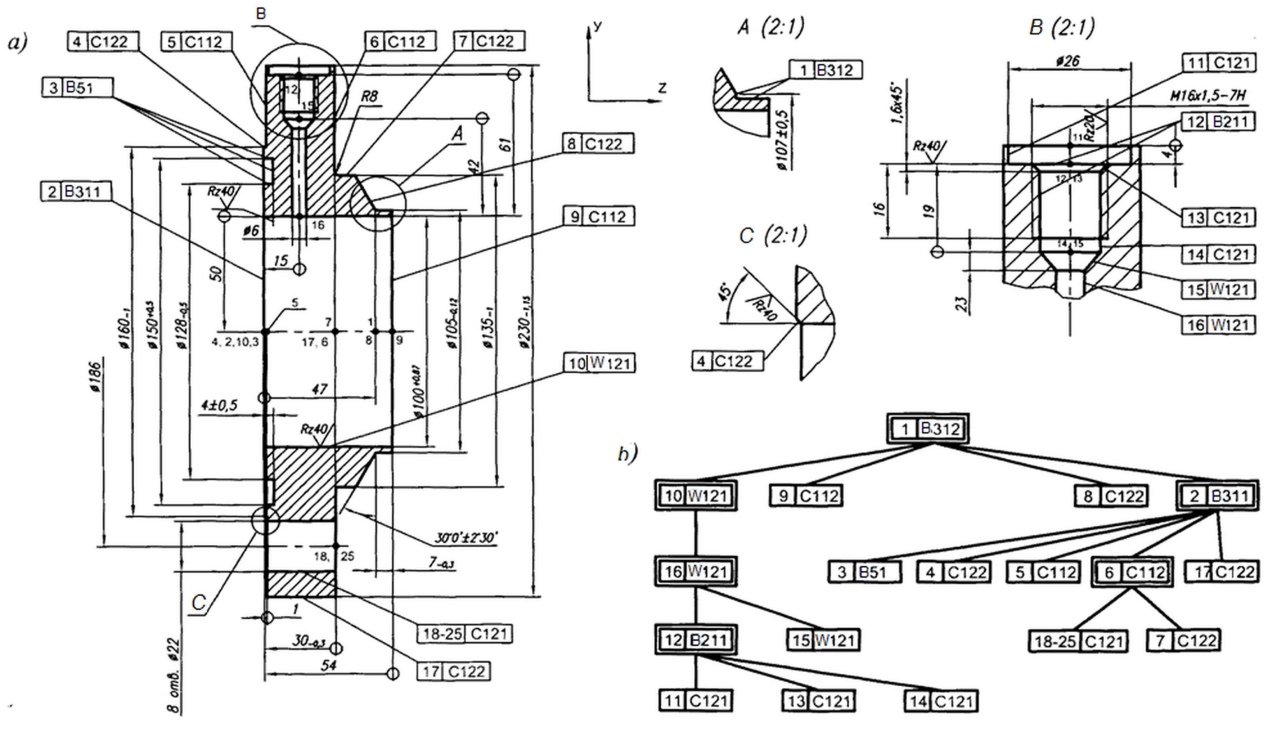

Fig. 3 Cover drawing by modular design (a) and part's graph (b)

The graph of the MSF details shows its structure at the modular level, th of the MSF, the level of complexity of the design. On the edges of the indicated tolerances on the coordinating dimensions of the MSF.

It is convenient to present information on the design characteristics of the form (Table 1).

It contains in sufficiently capacious form the MSF, their coordinatin. tolerances and design bases. Replacing the details presented in column 2 by $\varepsilon$.

MSF, we obtain the graph of a product of level 3 in the form of a structured set of MSF.

Table. 1. Part design specifications

\begin{tabular}{c|c|c|c|c|c|c|c|c}
\hline \multirow{2}{*}{$\begin{array}{c}\text { № } \\
\text { (fig.3) }\end{array}$} & \multirow{2}{*}{ MS } & \multirow{2}{*}{ Basing MS } & \multicolumn{5}{|c}{ Relative position parameters MS } \\
\cline { 5 - 9 } & & & $X, \mathrm{~mm}$ & $Y, \mathrm{~mm}$ & $Z, \mathrm{~mm}$ & $\varphi^{\circ}$ & $\psi^{\circ}$ & $\theta^{\circ}$ \\
\hline 1 & MSB 312 & - & 0 & 0 & 0 & 0 & 0 & 0 \\
\hline 2 & MSB 311 & 1 MSB 312 & 0 & 0 & -47 & 0 & 0 & 0 \\
\hline 3 & MSB 51 & 2 MSB 311 & 0 & 0 & 0 & 0 & 0 & 0 \\
\hline 4 & MSC 122 & 2 MSB 311 & 0 & 0 & 0 & 0 & 0 & 0 \\
\hline 5 & MSC 112 & 2 MSB 311 & 0 & 0 & 1 & 0 & 0 & 0 \\
\hline 6 & MSC 112 & 2 MSB 311 & 0 & 0 & $30-0,3$ & 0 & 0 & 0 \\
\hline 7 & MSC 122 & 1 MSB 312 & 0 & 0 & $-8,7$ & 0 & 0 & 0 \\
\hline 8 & MSC 122 & 1 MSB 312 & 0 & 0 & 0 & 0 & 0 & 0 \\
\hline 9 & MSC 112 & 2 MSB 311 & 0 & 0 & 54 & 0 & 0 & 0 \\
\hline 10 & MSW 121 & 1 MSB 312 & 0 & 0 & -47 & 0 & 0 & 0 \\
\hline
\end{tabular}




\begin{tabular}{c|c|c|c|r|r|r|r|r}
\hline 11 & MSC 121 & 12 MSB 211 & 0 & 4 & 0 & 0 & 0 & 0 \\
\hline 12 & MSB 211 & 12 MSW 121 & 0 & 61 & 0 & 0 & 0 & 0 \\
\hline 13 & MSC 121 & 12 MSB 211 & 0 & 0 & 0 & 0 & 0 & 0 \\
\hline 14 & MSC 121 & 12 MSB 211 & 0 & -19 & 0 & 0 & 0 & 0 \\
\hline 15 & MSW 121 & 16 MSW 121 & 0 & 42 & 0 & 0 & 0 & 0 \\
\hline 16 & MSW 121 & 10 MSW 121 & 90 & 50 & 15 & 0 & 0 & 0 \\
\hline 17 & MSC 122 & 1 MSB 312 & 0 & 0 & -17 & 0 & 0 & 0 \\
\hline 18 & MSB 311 & 1 MSB 312 & $-46,5$ & 80,4 & -17 & 0 & 0 & 0 \\
\hline 19 & MSB 311 & 1 MSB 312 & $-80,4$ & 46,5 & -17 & 0 & 0 & 0 \\
\hline 20 & MSB 311 & 1 MSB 312 & $-80,4$ & $-46,5$ & -17 & 0 & 0 & 0 \\
\hline 21 & MSB 311 & 1 MSB 312 & $-46,5$ & $-80,4$ & -17 & 0 & 0 & 0 \\
\hline 22 & MSB 311 & 1 MSB 312 & 46,5 & $-80,4$ & -17 & 0 & 0 & 0 \\
\hline 23 & MSB 311 & 1 MSB 312 & 80,4 & $-46,5$ & -17 & 0 & 0 & 0 \\
\hline 24 & MSB 311 & 1 MSB 312 & 80,4 & 46,5 & -17 & 0 & 0 & 0 \\
\hline 25 & MSB 311 & 1 MSB 312 & 46,5 & 80,4 & -17 & 0 & 0 & 0 \\
\hline
\end{tabular}

\section{Conclusion}

The various methods of classification, objects of unification allow, using the proposed coding, to carry out statistical processing on the parameters that are rational for a given production, taking into account the constraints laid down. This allows you to identify objects and their properties, most often used in the enterprise, and to form restrictive lists, the most effective for a particular production.

To improve the reliability of the system of technological preparation of production while reducing its adaptability, it is advisable to reduce the number of required positions and parameters to be optimized, and they will become restrictions. Restrictions can be set by examining the tools and determining their number or examining the structural elements of the part from the point of view of dimensions, accuracy and quality of surfaces, followed by determining the dimensions and accuracy class of the tools, their number in the set and on related operations.

The description of the structures of products by a hierarchical graph at the first level makes it possible to identify functional technological modules of the products and, on their basis, to construct a unified classification of products as objects of exploitation. Representation of parts by a set of modules allows to identify modules based, working and connecting surfaces and on their basis to build a single classification of parts, focused on different stages of the product life cycle.

The proposed unified methodological base allows you to manage the development of product designs, eliminate duplication in the creation of new designs and effectively develop technologies for their manufacture.

\section{References}

1. B.M. Bazrov, Modular technology in mechanical engineering (Moscow: Mashinostroenie, 2001) 
2. M.L. Kheifetz and B.P. Chemisov, Intellectual production: state and prospects (Novopolotsk: Polotsk State University, 2002)

3. A.S. Vasil'ev, S.A. Vasin, A.M. Dal'sky, A.I. Kondakov, Technological aspects of the conversion of engineering production in mechanical engineering (Moscow-Toula: Toula State University, 2003)

4. R. Hannam, Computer Integrated Manufacturing: from concepts to realization (Harlow: Addison Wesley, 1977)

5. Delchambke, Computer-aided Assembly Planning (London: Chapman \& Hall, 1992)

6. P. Groche, E. Bruder, S. Gramlich, Manufacturing Integrated Design: Sheet Metal Product and Process Innovation (N.Y.: Springer, 2017)

7. R. Light, D. Gossard, CAD, V.14, 84, 209 (1982)

8. G. Spur, O. Kurz, ZWP, 3, 130 (1982)

9. J. Gausemeier, ZWF, 9, 467 (1986)

10. D.T. Ross, R.E. Schoman, IEEE Transaction on SE, V. SE, 1, 6 (1997)

11. B.I. Cherpakov, Computerized integrated production and CALS-technologies in mechanical engineering (Moscow, State Unitary Enterprise "VIMI", 1999)

12. A.G. Bratukhin, CALS in aircraft construction (Moscow: Moscow Aviation Institute, 2000)

13. H.A. Youssef, H.A. El-Hofy, M.H. Ahmed, Manufacturing Technology: Materials, Processes, and Eqipment (N.Y.: CRC Press - Taylor, 2017)

14. A.M. Rusetsky, Theoretical bases of technological systems designing (Minsk: Belaruskaya Navuka, 2012)

15. A.M. Rusetsky, Design and equipment of technological systems (Minsk: Belaruskaya Navuka, 2014)

16. B.M. Bazrov, Engineering Proceedings, 2, 51 (2011) 